\title{
Emergence of a peak in early infant mortality due to HIV/AIDS in South Africa
}

\author{
David E. Bourne ${ }^{a}$, MaryLou Thompson ${ }^{a, b}$, Linnea L. Brody ${ }^{a, f}$, \\ Mark Cotton', Beverly Draper ${ }^{\mathrm{a}}$, Ria Laubscher', \\ M. Fareed Abdullah ${ }^{\mathrm{e}}$ and Jonny E. Myers ${ }^{\mathrm{a}}$
}

\begin{abstract}
Objectives: South Africa has among the highest levels of HIV prevalence in the world. Our objectives are to describe the distribution of South African infant and child mortality by age at fine resolution, to identify any trends over recent time and to examine these trends for HIV-associated and non HIV-associated causes of mortality.

Methods: A retrospective review of vital registration data was conducted. All registered postneonatal deaths under 1 year of age in South Africa for the period 1997-2002 were analysed by age in months using a generalized linear model with a log link and Poisson family.

Results: Postneonatal mortality increased each year over the period 1997-2002. A peak in HIV-related deaths was observed, centred at 2-3 months of age, rising monotonically over time.

Conclusion: We interpret the peak in mortality at 2-3 months as an indicator for paediatric AIDS in a South African population with high HIV prevalence and where other causes of death are not sufficiently high to mask HIV effects. Intrauterine and intrapartum infection may contribute to this peak. It is potentially a useful surveillance tool, not requiring an exact cause of death. The findings also illustrate the need for early treatment of mother and child in settings with very high HIV prevalence.
\end{abstract}

๑ 2009 Wolters Kluwer Health | Lippincott Williams \& Wilkins

AIDS 2009, 23:101-106

Keywords: demography, mortality, paediatric AIDS, postneonatal, South Africa

\section{Introduction}

South Africa is currently experiencing a severe HIV/AIDS epidemic, with corresponding high infant mortality. The mean infant mortality rate in South Africa for the period 1993-1998 was 45.4, which is rather low compared with other sub-Saharan African countries [1]. The prevalence of HIV infection among women attending antenatal clinics in the public sector was estimated to be $7.6 \%$ in 1994, increasing to $22.8 \%$ in 1998 , and peaking at $30.3 \%$ by 2005 [2]. Prevention of mother-to-child transmission programmes were introduced gradually into South Africa from 2001, initially in the Western Cape Province [3-5]. Antiretroviral (ARV) medication was not generally available in public sector facilities in most of South Africa until as late as 2004. In the absence of intervention measures, it is estimated that the proportion of infants infected with HIV through mother-to-child transmission is between 25 and $45 \%$ [6]. In an early study from the KwaZulu-Natal Province conducted in 1992, the transmission proportion was 34\% [7], and in utero infection, in an era before ARV administration, has been reported as $27 \%$ [8]. Our objectives are to describe the distribution of infant and child mortality by age in months and to identify any

\footnotetext{
${ }^{a}$ School of Public Health and Family Medicine, University of Cape Town, Cape Town, South Africa, ${ }^{b}$ Department of Biostatistics, University of Washington, Seattle, USA, ${ }^{\mathrm{C}}$ Department of Paediatrics and Child Health, Stellenbosch University, South Africa, ${ }^{\mathrm{d} B i o s t a t i s t i c s}$ Unit, South African Medical Research Council, Cape Town, South Africa, ${ }^{\mathrm{e}}$ The Global Fund to Fight AIDS, Tuberculosis and Malaria, Geneva, Switzerland, and ${ }^{\mathrm{f} B u r d e n}$ of Disease Research Unit, South African Medical Research Council, Cape Town, South Africa

Correspondence to David E. Bourne, School of Public Health and Family Medicine, University of Cape Town, Cape Town 7505, South Africa.
}

E-mail: David.Bourne@uct.ac.za

Received: 25 April 2008; revised: 17 September 2008; accepted: 29 September 2008.

DOI:10.1097/QAD.0b013e32831c54bd

ISSN 0269-9370 @ 2009 Wolters Kluwer Health | Lippincott Williams \& Wilkins 
trends over recent time. We assess trends in HIV-associated and non HIV-associated causes of mortality. South Africa provides a unique environment to examine these objectives, as there is a combination of an endemic high prevalence of HIV and the availability of a relatively sophisticated vital statistics system.

\section{Methods}

\section{Data sources}

We obtained South African infant mortality counts by age in months under 5 years for the period 1997-2002 as a special tabulation from Statistics South Africa (STATS SA), including underlying cause of death, coded to the International Classification of Diseases (ICD-10) at the three-character level [9]. Although STATS SA data are coded to three characters of the ICD, this does not necessarily mean that these diagnoses are correct at this level. In fact, there is substantial misclassification at this apparent level of detail, and only the broadest of categories (the Chapter codes) will retain any validity [10]. On the basis of ICD-10 Chapter codes, we divided all postneonatal deaths under 1 year of age into two groups - those likely to be, and those not likely to be, caused by an HIV infection in the child (Table 1). Chapter codes in the group unlikely to be related to HIV/AIDS are least liable to be subject to misclassification. The group likely to be related to HIV/AIDS contains all the other Chapter codes and is more liable to misclassification. It includes HIV/AIDS as a reported cause of death as well as perinatal (Chapter XVI) and unclassified (Chapter XVIII) causes. HIV/AIDS as a reported cause of death is subject to serious underreporting in South Africa [11]. We believe that significant numbers of the deaths recorded in the unclassified and perinatal categories will, in fact, be HIV-related. Unclassified deaths constitute a small proportion, $2 \%$ on average, of all postneonatal deaths, whereas on average $76 \%$ of deaths at $1-11$ months were attributed to perinatal causes. STATS SA has acknowledged misclassification in the perinatal Chapter [12]. Although there is potential for misclassification in this grouping, this can only serve to dilute any HIVrelated trends in the data.

Table 1. Causes of infant death (the International Classification of Diseases-10 Chapter categories) likely and unlikely to be associated with HIV/AIDS.

\begin{tabular}{|c|c|}
\hline $\begin{array}{l}\text { Likely HIV/AIDS-associated } \\
\text { causes }\end{array}$ & $\begin{array}{l}\text { Causes unlikely to be associated } \\
\text { with HIV/AIDS }\end{array}$ \\
\hline $\begin{array}{l}\text { Infectious/parasitic } \\
\text { Blood/immune } \\
\text { Endocrine/nutritional/metabolic } \\
\text { Circulatory } \\
\text { Respiratory } \\
\text { Digestive } \\
\text { Perinatal conditions } \\
\text { Unclassified }\end{array}$ & $\begin{array}{l}\text { Neoplasms } \\
\text { External causes (non-natural) } \\
\text { Congenital } \\
\text { Genitourinary } \\
\text { Skin } \\
\text { Musculo/connective tissue } \\
\text { Mental } \\
\text { Nervous } \\
\text { Ear }\end{array}$ \\
\hline
\end{tabular}

Deaths recorded before age 1 month were excluded from analyses because of the potential misclassification of live and stillbirths. During the time period under study, the number of births remained approximately static at about 1.1 million per annum [13].

\section{Statistical analysis}

The following generalized linear model (GLM) with a log link and Poisson family [14] was used to model postneonatal HIV/AIDS-associated mortality counts under 1 year of age for the period 1997-2002:

$\ln \left(E\left(Y_{\mathrm{ij}}\right)\right)=\alpha_{0}+\beta_{1} j+\beta_{2} M+\gamma_{1} i+\gamma_{2} R$

where $Y_{\mathrm{ij}}$ is the number of deaths at age $j$ months in year $i$; $M=(j-2.5)$ for $j \geq 2.5, M=0$ otherwise; $i=0$ for 1997 , $i=1$ for 1998 and so on; $R=i$ if age $=2-3$ months, $R=0$ otherwise. For a given year, this models the annual age-specific mortality rate (on the log scale) by a linear spline in age with a knot at 2.5 months of age, that is, by two straight lines with possibly different slope before $\left(\beta_{1}\right)$ and after $\left(\beta_{1}+\beta_{2}\right) 2.5$ months of age. The mortality trend over years at each age is modelled separately for age at death $2-3$ months $\left(\gamma_{1}+\gamma_{2}\right)$ and other ages $\left(\gamma_{1}\right)$. Hence the null hypothesis $H_{0}: \beta_{2}=0$ assesses whether there is a change at $2-3$ months in the trend in age-specific mortality within a given year. The null hypothesis $H_{0}$ : $\gamma_{2}=0$ assesses whether the change in mortality over time at ages 2-3 months is different from those at other ages. Robust variance estimation [15] was used to accommodate correlation in the mortality counts and possible deviations from the Poisson mean-variance structure. $Z$-statistics (parameters divided by their robust standard errors) were used to test the above hypotheses. Statistical significance was defined as $P$ value less than 0.05. Analyses were carried out using Stata statistical software, Version $9.1[16]$.

\section{Results}

Figure 1 shows the changing mortality pattern from birth to 5 years of age for the period 1997-2002. Clearly evident is the high neonatal mortality, an emerging peak of mortality centred at ages $2-3$ months, and thereafter a decline in mortality up to 5 years of age. In 1997, there were 11469 recorded neonatal deaths in South Africa before age 1 month, representing $43.8 \%$ of South African deaths within the first year of life. By 2002, these had increased in absolute terms to 12152 recorded infant deaths before age 1 month, when neonatal deaths constituted only $33.3 \%$ of 2002 deaths within the first year of life. However, between 1997 and 2002, deaths at $2-3$ months of age increased from 30.5 to $35.5 \%$ of the postneonatal mortality within the first year of life. In 1997, there were 7322 deaths at between 1 and 5 years of age; by 2002, this had increased to 11917 deaths. 


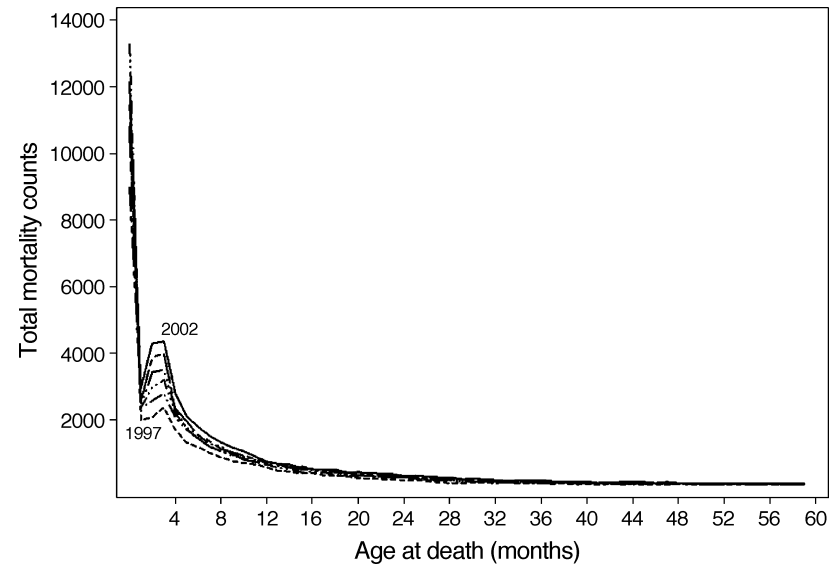

Fig. 1. Graphical representation of total deaths, at ages 0-60 months, in South Africa during 1997-2002.

An increase over time in the number of postneonatal infant deaths likely to be associated with HIV/AIDS at all ages under 1 year is apparent (Fig. 2), with a more marked increase at ages $2-3$ months. Table 2 shows the estimated mortality rate ratios corresponding to the fitted generalized linear model. The GLM analysis indicated that, in each calendar year, expected mortality increased significantly with increasing age of death up to 2.5 months and then decreased significantly thereafter, that is, a peak in mortality exists at age $2-3$ months. The null hypothesis that there is no change in trend in mortality after $2-3$ months $\left(H_{0}: \beta_{2}=0\right)$ is rejected $(P<0.0005)$. Further, the rate of increase in mortality from year to year at ages $2-3$ months was significantly greater than that at other ages of death $\left(H_{0}: \gamma_{2}=0\right.$ is rejected, $\left.P<0.0005\right)$, that is, the peak was accelerating. A more richly parameterized model with main effects for year and age (in months) provided a better fit to the data (analysis not shown). However, the conclusions from the model summarized in Table 2, which has the advantages of parsimony and ease of interpretation, are entirely consistent with those for the more complex model.

Postneonatal infant mortality counts not associated with HIV/AIDS remained relatively stable over the time period under consideration and showed no indication of a peak at age 2-3 months (Fig. 3). Mortality across age of death within each calendar year showed a significant

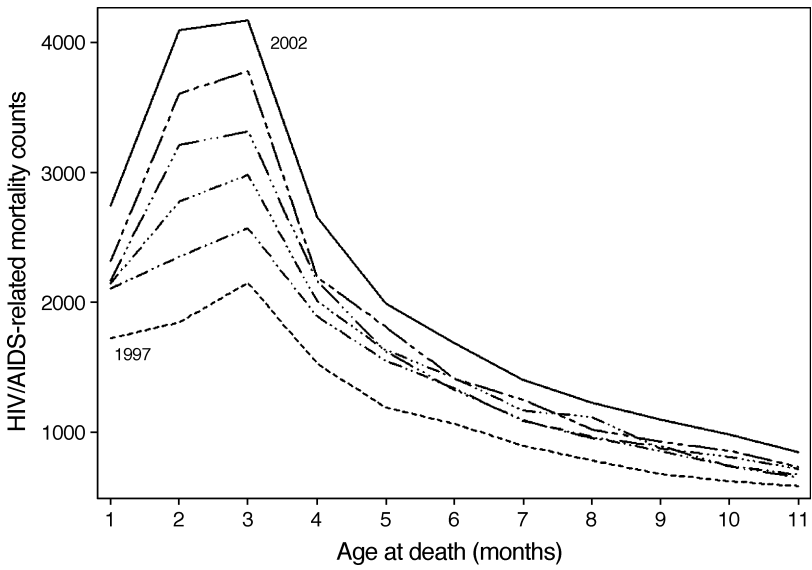

Fig. 2. Graphical representation of likely HIV/AIDS-related deaths, at ages 1-11 months, in South Africa during 19972002.

decline from age 1 month $(P<0.0005)$, with no change in that downward trend at $2-3$ months of age $(P=0.57)$.

\section{Discussion}

The typical mortality pattern by age during the first year of life shows a decline with increasing age. We have, however, observed the emergence of a substantial and monotonically increasing peak in the South African population all-cause mortality during the early postneonatal period, as well as in those deaths, which are more likely to be associated with HIV. For deaths unlikely to be associated with HIV/AIDS, there was no peak in the early postneonatal period, nor any increase over time, indicating that an increase in registration efficiency of infant deaths was not an artefactual cause of the overall observed peak in deaths. In addition, it does not seem plausible that increases in registration over time could lead to recorded mortality at ages $2-3$ months increasing at a greater rate than the recorded mortality at other months of age. Although we acknowledge that the ICD-10 codes that we have grouped to represent HIV/AIDS-related causes of death are subject to misclassification, we nevertheless believe that this misclassification would only serve to dilute any HIV/AIDS-related trends in mortality. In support of this contention, we have carried out

Table 2. Estimated mortality rate ratios from generalized linear model at ages 1-11 months during 1997-2002.

\begin{tabular}{llr}
\hline & Estimated mortality rate ratio $(95 \% \mathrm{Cl})$ & $P$-value \\
\hline Within a calendar year, corresponding to 1 month increase in age: & & \\
Prior to 2.5 months of age $\left(e^{\beta_{1}}\right)$ & $1.12(1.07,1.17)$ & $<0.0005$ \\
After 2.5 months of age $\left(e^{\beta_{1}+\beta_{2}}\right)$ & $0.85(0.84,0.86)$ & $<0.0005$ \\
At a given age, comparing successive years: & $1.07(1.06,1.09)$ & $<0.0005$ \\
Age $\neq 2-3$ months $\left(e^{\gamma_{1}}\right)$ & $1.16(1.15,1.18)$ & $<0.0005$ \\
Age $=2-3$ months $\left(e^{\gamma_{1}+\gamma_{2}}\right)$ & \\
\hline
\end{tabular}

$\mathrm{Cl}$, confidence interval. 


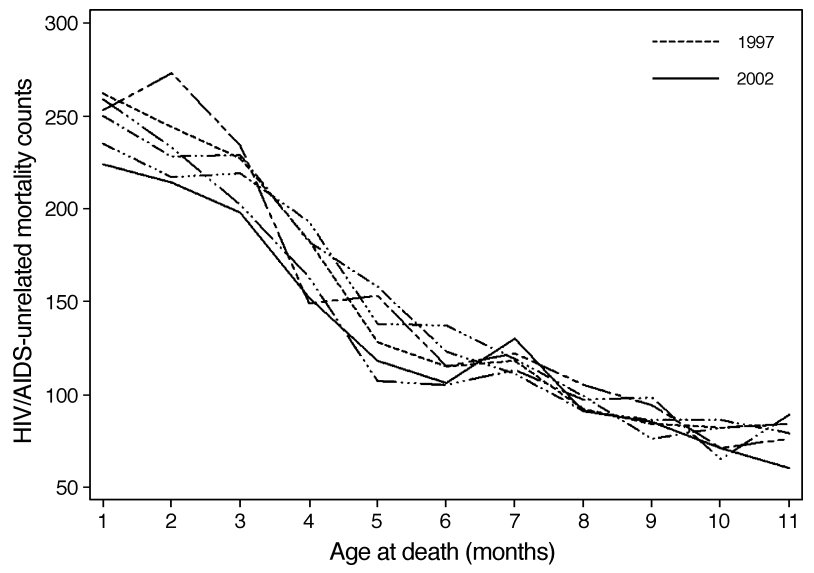

Fig. 3. Graphical representation of deaths unlikely to be related to HIV/AIDS, at ages 1-11 months in South Africa during 1997-2002.

separate analyses for two HIV/AIDS-related ICD-10 Chapter codes, which are unlikely to be subject to misclassification: infectious and parasitic diseases (Chapter I) and diseases of the blood and immune mechanism (Chapter III). In these data, we observe an even more pronounced increasing peak in mortality at ages $2-3$ months (data not shown). These observations constitute convincing evidence for HIV/AIDS causation.

Although we did not a priori anticipate a peak in postneonatal mortality at ages $2-3$ months, the consistent presence of this peak in each year over the period under consideration makes it extremely unlikely to be a chance finding. Newell et al. [17] previously documented cumulative mortality of HIV-infected and uninfected infants born to HIV-infected mothers in Africa. Although they did not discern this peak, our results led us to transform their estimated cumulative mortality estimates into age-specific mortality probabilities, which showed a similar peak at about 4 months of age. To our knowledge, there are no other reports in the literature relating to this peak in early infant mortality in settings of high HIV prevalence.

The magnitude of this peak in mortality at the population level is substantial. It is also notable that no other country experiencing a severe AIDS epidemic has been capable of analysing infant mortality at this fine age resolution. If traditional child mortality indicators such as neonatal and infant mortality had been used, the observed peak would have been missed. Cohort studies have also focussed on conventional summary measures such as cumulative mortality as opposed to age-specific hazards.

Although such a peak in mortality is unlikely to be detected in poorly resourced settings without a relatively sophisticated vital registration system [18], it is also unlikely to arise in well resourced settings with low HIV prevalence and good healthcare, where the number of deaths would be too small to discern such a pattern. In the whole of North America and Western Europe, there is approximately one case of vertical transmission of HIV daily compared with 1700 in sub-Saharan Africa [19]. It still needs to be shown whether this peak will emerge in other less developed settings with extremely high infant mortality.

There is some local epidemiological evidence for increased mortality due to HIV/AIDS during the first 2 years of life compared with the period from 2 to 5 years of age. The earlier group is thought to have become infected in utero or during delivery, whereas the latter is thought to become infected through breastfeeding [20]. A healthy mother provides maternal immunity to her infant through placental transfer and through breast milk. By approximately 3 months after birth, an infant's maternally acquired immunity is in decline, whereas the infant's own immunity, which begins development in utero, is still very immature [21]. In the presence of in-utero HIV infection, the infant's immunity is likely to be further compromised.

Studies [22-24] have also shown that infants born to mothers who are HIV positive receive deficient maternal immunity as a result of deficient placental transfer of maternal antibodies, or because an HIV-positive mother may simply have levels of antibodies too low for sufficient transfer. Further, studies [25,26] suggest that an HIVinduced deficient maternal immune system fails to adequately support the development of a competent immune system in infants. Studies have also shown an increase in mortality in HIV-uninfected infants born to HIV-positive mothers [27]. Kuhn et al. [24] have linked this with advanced maternal disease in a Zambian cohort.

Further evidence indicating immunological immaturity in neonates has been shown for nef-deleted SIV infection, which, while not virulent in adult macaques, is highly pathogenic in neonatal macaques [28]. Factors permitting high viral replication in infants include a larger pool of $\mathrm{CD} 4^{+}$T-cells, a large thymus, delayed development of cytotoxic lymphocyte activity, and antibody-dependent cytotoxicity [29]. Infants cannot suppress HIV replication as effectively as adults. In a prospective study comparing newly infected Kenyan adults and infants, the mean peak HIV-1 RNA was significantly higher in infants than adults, as was the mean set point level, indicating poorer ability to control HIV replication in infants. Further evidence is that the viral set point in infants infected later in life through breastfeeding was far lower than that in infants infected earlier [30]. Evidence that early initiation of ARV therapy (by 6 weeks of age) reduces mortality by $75 \%$ compared with waiting for immunological markers to be below threshold values [31] corroborates the interpretation from this study that infants born infected with HIV are at particular risk of dying a few months after birth. 
In the present study, the sharp increase in mortality seen at 2-3 months is likely to be a result of the particular immunological vulnerability of HIV-infected neonates at this age. The most likely event that causes the peak is the loss of acquired maternal immunity at 2-3 months in children who have either been infected in utero or intrapartum. Another explanation could be acute retroviral syndrome from intrapartum infection, which would manifest from approx 6 weeks of age - this has been described in African infants [32].

By contrast, acquisition of HIV infection from breast milk is likely to take place over an extended period of time, as will the manifestation of the sequelae of infection leading to death. We hypothesize that this particular mechanism will not lead to a sharp peak of mortality, but is more likely to lead to an extended tail of mortality beyond the peak described here.

In populations similar to those of South Africa with high HIV prevalence and where other causes of death are not sufficiently high to mask HIV effects, the observed peak in mortality in early infancy, which is a new demographic phenomenon, provides a potentially useful surveillance tool for monitoring paediatric AIDS without requiring explicitly diagnosed cause of death.

\section{Acknowledgements}

D.B. is in receipt of a self-initiated research grant from the SA Medical Research Council. The University of Cape Town health sciences faculty also provided partial support, as did the Burden of Disease research project of the Western Cape Department of Health. We thank Dr Heston Philips of Statistics South Africa for producing the special tabulations for 1997-2002, Dr Marie-Louise Newell from the Africa Centre for sharing data on cumulative mortality, and $\operatorname{Dr} A$ Boulle for useful comments. We are appreciative of helpful comments from two anonymous reviewers.

D.B. is the primary author who conceived and designed the study, directed its implementation, and carried out preliminary analyses with L.B. and R.L. L.B., an MPH student, was also responsible for data management. M.L.T. provided biostatistical consultation, devised and conducted advanced statistical analyses, and provided critical review. B.D. and F.A. contributed to the health systems, M.C. to the clinical and J.M. to the epidemiological aspects of the interpretation of findings. All authors collectively contributed to drafting and editing of the article.

Ethics approval was obtained from the University of Cape Town Research Ethics Committee REC/REF 071/ 2008. There are no conflicts of interest.

\section{References}

1. Department of Health, Medical Research Council, Measure DHS+. South Africa Demographic and Health Survey 1998. Full Report. Pretoria: Department of Health; 2002.

2. Department of Health. National HIV and syphilis antenatal seroprevalence survey in South Africa 2006. Pretoria: Department of Health; 2007.

3. Doherty T, Besser M, Donohue S, Kamoga N, Stoops N, Williamson L, Visser R. Case Study Reports on Implementation and Expansion of the PMTCT Programme in the Nine Provinces of South Africa. Durban: Health Systems Trust; 2003.

4. Abdullah MF, Young T, Bitalo L, Coetzee N, Myers JE. Public health lessons from a pilot programme to reduce mother-tochild transmission of HIV-1 in Khayalitsha. S Afr Med / 2001; 81:579-583.

5. Eley B. Addressing the paediatric HIV epidemic: a perspective from the Western Cape Region of South Africa. Trans $R$ SoC Trop Med Hyg 2006; 100:19-23.

6. The Working Group on Mother-to-Child Transmission of HIV. Rates of mother-to-child transmission of HIV-1 in Africa, America and Europe: results from 13 perinatal studies. J Acquir Immune Defic Syndr Hum Retrovirol 1995; 8:506510.

7. Bobat R, Coovadia H, Coutsoudis A, Moodley D. Determinants of mother-to-child transmission of human immunodeficiency virus type 1 infection in a cohort from Durban, South Africa. Pediatr Infect Dis / 1996; 15:604-610.

8. Magder LS, Mofenson L, Paul ME, Zorrilla CD, Blattner WA, Tuomala RE, et al. Risk factors for in utero and intrapartum transmission of HIV. I Acquir Immune Defic Syndr 2005; 38: 87-95.

9. WHO. International statistical classification of diseases and health related problems. Tenth revision. Geneva: World Health Organization; 1992.

10. Brody LL. Infant and child mortality in South Africa in the context of a high HIV prevalence: an investigation into changing mortality patterns at a fine age resolution. MPH Thesis. University of Cape Town; 2007.

11. Groenewald P, Nannan N, Bourne D, Laubscher R, Bradshaw D. Identifying deaths from AIDS in South Africa. AIDS 2005; 19:193-201.

12. Statistics South Africa. Mortality and causes of death in South Africa, 2005: findings from death notification. Statistical release P0309.3. Pretoria: Statistics South Africa; 2007.

13. Moultrie TA, Dorrington R. Estimation of fertility from the 2001 South Africa Census data. CARe Monograph no. 12. Centre for Actuarial Research University of Cape Town; 2004.

14. McCullagh P, Nelder JA. Generalized linear models. London: Chapman and Hall; 1989.

15. Williams RL. A note on robust variance estimation for clustercorrelated data. Biometrics 2000; 56:645-646.

16. Stata Corp. Stata Statistical Software, Release 9.1. College Station, TX: Stata Corporation; 2005.

17. Newell ML, Coovadia H, Cortina-Borja M, Rollins N, Gaillard P, Dabis F. Mortality of infected and uninfected infants born to HIV-infected mothers in Africa: a pooled analysis. Lancet 2004; 364:1236-1243.

18. Setel PW, Macfarlane SB, Szreter S, Mikkelsen L, Jha P, Stout S, et al. A scandal of invisibility: making everyone count by counting everyone. Lancet 2007; 370:1569-1577.

19. McIntyre J. Preventing mother-to-child transmission of HIV: successes and challenges. BJOG 2005; 112:1196-1203.

20. Luzuriaga K, Sullivan JL. Viral and immunopathogenesis of vertical HIV-1 infection. Pediatr Clin North Am 2000; 47:6578.

21. Zinkernagel RM. Maternal antibodies, childhood infections, and autoimmune diseases: advances in immunology. $N$ Eng/ / Med 2001; 345:1331-1335.

22. de Moraes-Pinto MI, Farhat CK, Carbonare SB, Curti SP, Otsubo ME, Lazarotti DS, et al. Maternally acquired immunity in newborns from women infected by the human immunodeficiency virus. Acta Paediatr 1993; 82:1034-1038.

23. de Moraes-Pinto MI, Almeida AC, Kenj G, Filgueiras TE, Tobias $\mathrm{W}$, Santos $\mathrm{AM}$, et al. Placental transfer and maternally acquired neonatal IgG immunity in human immunodeficiency virus infection. J Infect Dis 1996; 173:1077-1084. 
24. Kuhn L, Kasonde P, Sinkala M, Kankasa C, Semrau K, Scott N, et al. Does severity of HIV disease in HIV-infected mothers affect mortality and morbidity among their uninfected infants? Clin Infect Dis 2005; 41:1654-1661.

25. Chougnet C, Kovacs A, Baker R, Mueller B, Luban N, Liewehr D, et al. Influence of human immuno-deficiency virus-infected maternal environment on development of infant interleukin-12 production. I Infect Dis 2000; 181:1590-1597.

26. Clerici M, Saresella M, Colombo F, Fossati S, Sala N, Bricalli D, et al. T-lymphocyte maturation abnormalities in uninfected newborns and children with vertical exposure to HIV. Blood 2000; 96:3866-3871.

27. Graham SM, Gibb DM. HIV disease and respiratory infection in children. British Medical Bulletin 2002; 61:133-150.

28. Baba TW, Jeong YS, Pennick D, Bronson R, Greene MF, Ruprecht RM. Pathogenicity of live, attenuated SIV after mucosal infection of neonatal macaques. Science 1995; 267:1820-1825.
29. Richardson BA, Mbori-Ngacha D, Lavreys L, John-Stewart GC, Nduati R, Panteleeff DD, et al. Comparison of human immunodeficiency virus type 1 viral loads in Kenyan women, men, and infants during primary and early infection. J Viro/ 2003; 77:7120-7123.

30. Spira R, Lepage P, Msellati P, Van De Perre P, Leroy V, Simonon $A$, et al. Natural history of human immunodeficiency virus type 1 infection in children: a five-year prospective study in Rwanda. Pediatrics 1999; 104:e56.

31. Violari A, Cotton MF, Gibb DM, Babiker AG, Steyn M, Madhi SA, et al. Early Antiretroviral Therapy and Mortality among HIV-Infected Infants. N Engl I Med 2008; 359: 2233-2244.

32. Richardson BA, Nduati N, Mbori-Ngacha D, Overbaugh J, JohnStewart GC. Acute HIV infection among Kenyan infants. Clin Infect Dis 2008; 46:289-295. 